\title{
Rapid, direct detection of bacterial Topoisomerase 1-DNA adducts by RADAR/ELISA
}

\author{
Devapriya Sinha ${ }^{1, *}$, Kostantin Kiianitsa ${ }^{1, *}$, David R. Sherman ${ }^{2}$, Nancy Maizels ${ }^{1,3}$ \\ ${ }^{1}$ Department of Immunology, University of Washington, 1959 NE Pacific St., Seattle, \\ WA 98195, USA \\ ${ }^{2}$ Department of Microbiology, University of Washington, 815 Republican St., Seattle, \\ WA 98102, USA \\ ${ }^{3}$ Department of Biochemistry, University of Washington, 1959 NE Pacific St., Seattle, \\ WA 98195, USA
}

*The authors wish it to be known that, in their opinion, the first two authors should be regarded as Joint First Authors.

To whom correspondence should be addressed. Tel., +1 206-685-4449; Fax: +1 206221-6781; Email: kkiianit@uw.edu

Running title: Direct Assay of Bacterial Top1-DNA Adducts

Keywords: DNA-protein crosslink, gyrase, Mycobacteria, tuberculosis, antibiotic, topoisomerase poison

\footnotetext{
1 Abstract

2 Topoisomerases are proven drug targets, but antibiotics that poison bacterial

3 Topoisomerase 1 (Top1) have yet to be discovered. We have developed a rapid and

4 direct assay for quantification of Top1-DNA adducts that is suitable for high throughput

5 assays. Adducts are recovered by "RADAR fractionation", a quick, convenient

6 approach in which cells are lysed in chaotropic salts and detergent and nucleic acids

7 and covalently bound adducts then precipitated with alcohol. Here we show that

8 RADAR fractionation followed by ELISA immunodetection can quantify adducts formed

9 by wild-type and mutant Top1 derivatives encoded by two different bacterial pathogens,

10 Y. pestis and M. tuberculosis, expressed in E. coli or M. smegmatis, respectively. For

11 both enzymes, quantification of adducts by RADAR/ELISA produces results comparable

12 to the more cumbersome classical approach of $\mathrm{CsCl}$ density gradient fractionation. The

13 experiments reported here establish that RADAR/ELISA assay offers a simple way to

14 characterize Top1 mutants and analyze kinetics of adduct formation and repair. They
} 
15 also provide a foundation for discovery and optimization of drugs that poison bacterial

16 Top1 using standard high-throughput approaches.

\section{INTRODUCTION}

19 There is a widely recognized need to develop new drugs to treat infections, especially as many microorganisms have developed resistance to antibiotics in common use. Topoisomerases have proven to be effective drug targets not only in infectious disease but also in cancer $(1,2)$. Topoisomerases modulate DNA topology by catalyzing cleavage and then religation of one or both strands of the duplex, forming a covalent topoisomerase-DNA complex as an obligatory reaction intermediate (3) (Fig. 1A). Accumulation of toxic Top1-DNA covalent intermediates contributes to killing of cells in which drugs or mutations impair the normal Top1 reaction cycle. Drugs that target topoisomerases cause the covalent adduct to persist, preventing release of the bound protein and religation of the DNA. This is the mechanism of cell killing by fluoroquinolone antibiotics, such as ciprofloxacin, moxifloxacin, levofloxacin, and ofloxacin, which target DNA gyrase (4); and of chemotherapeutics used to treat cancer, such as topotecan and etoposide $(5,6)$. Mutations in topoisomerases can also impair their ability to release the covalent bond and reseal the DNA duplex.

The only bacterial topoisomerases targeted by drugs to date are the Type II topoisomerases, gyrase and topoisomerase IV. Efforts to identify potent small molecule inhibitors of bacterial Type IA enzymes have had only limited success thus far (7). Key to the religation step catalyzed by bacterial Top 1 is coordination of a metal ion by aspartate residues of an acidic triad within the DxDxxG motif of the highly conserved Top1 TOPRIM domain (8,9) (Fig. 1B). Mutational analysis has shown that modification of the $\mathrm{N}$ - or $\mathrm{C}$-terminal residues of this domain in Top1 from bacterial species, including Escherichia coli, Yersinia pestis, Mycobacterium tuberculosis and Mycobacterium smegmatis, will impair DNA religation, induce an SOS response and cause cell killing (10-14).

The absence of a simple, mechanism-based assay that quantifies Top1-DNA adducts formed in vivo has been a stumbling block to drug discovery and development for this target. Some features of topoisomerases themselves contribute to difficulty in systematic detection. Topoisomerase-DNA adducts are normally transient and may resolve spontaneously when drug is removed or limiting; cells may lyse in response to cell killing, releasing adducted complexes into the culture medium. The adducted enzyme may also undergo proteolytic repair, eliminating epitopes for immunodetection, as we recently demonstrated for human endogenous topoisomerase-DNA adducts by an unbiased proteomic approach (15). As the first step toward overcoming these challenges, we have developed a rapid and direct assay for quantification of topoisomerase-DNA adducts formed in living cells. The assay is based on a method referred to as "RADAR" fractionation $(16,17)$, in which cells are lysed in chaotropic salts 
and detergent; nucleic acids and adducted proteins are separated from free protein by alcohol precipitation; and adducted protein is quantified by immunodetection. RADAR fractionation has enabled quantification of adducts formed by bacterial and mammalian topoisomerases (16-23) and by Pol beta (24) and other mammalian proteins $(21,25)$. We have recently validated the ability of RADAR fractionation to enrich adduct-forming proteins from human cells by mass spectrometry (15). RADAR fractionation is costand time-effective, and provides a considerable improvement in throughput (100-fold or more) over the classical approach for adduct recovery by $\mathrm{CsCl}$ density gradient fractionation (26).

Here we describe an assay for bacterial Top1-DNA adducts that combines RADAR fractionation and ELISA assay in microplate format. We have developed this assay using as a model Top1-DNA adducts encoded by two different bacterial pathogens, $Y$. pestis and $M$. tuberculosis, expressing epitope-tagged Top1 from inducible constructs. This enables detection by highly specific commercially available antibodies, circumventing the need to identify and characterize antibodies specific to each target of interest. We apply the RADAR/ELISA assay to quantify accumulation of DNA adducts formed by the highly toxic Y. pestis mutant, YpTop1-D117N, expressed in E. coli cells. The Z' factor of the assay is $>0.5$, suitable for high throughput applications. We further demonstrate that RADAR lysis disrupts the normally challenging mycobacteria cell wall and enables recovery and quantification of adducts formed by MtTop1-DNA expressed in $M$. smegmatis. These experiments provide a foundation for discovery and optimization of drugs that poison bacterial Top1 using standard highthroughput approaches.

\section{MATERIALS AND METHODS}

\section{Cloning and site-directed mutagenesis of MtTop1 constructs}

Gateway LR clonase (Thermo-Fisher) was used to clone the MtTop1 coding sequence from the pENTR vector ( $p E N T R: 3646 c ;(27)$ ) into the pDTNF expression vector (pDTNF:3646c), fusing a FLAG tag to the N-terminal of Top1. The D111A, D111N, and G116S mutants of MtTop1 were generated by QuikChange using Pfu Turbo (Thermo-

87 Fisher).

\section{Cell culture, Top1 induction, and viability assays} YpTop1-D117N (BW117N) bearing an N-terminal TRX tag was a gift from Prof Yuk-

93 Ching Tse-Dinh (Florida International University, USA). Cells were cultured at $37^{\circ} \mathrm{C}$ in

94 LB medium containing $25 \mu \mathrm{g} / \mathrm{ml}$ chloramphenicol. Top1 expression was induced in 
95 exponentially growing cultures $\left(\mathrm{OD}_{600}=0.3\right)$ by addition of arabinose (Sigma Aldrich).

96

97

98

99

100

101

102

103

104

105

106

107

108

109

110

111

112

113

114

115

116

117

118

119

120

121

122

123

124

125

126

127

128

129

130

131

132

133

134

Cell division was monitored by measuring $\mathrm{OD}_{600}$.

M. smegmatis ( $\mathrm{mc}^{2155}$ ) strain was cultured in $7 \mathrm{H} 9$ medium with ADC supplement (HiMedia) as described (28). M. smegmatis was transformed using a MicroPulser ${ }^{\mathrm{TM}}$ Electroporator Bio-Rad), and transformed cells selected and propagated in medium containing $200 \mu \mathrm{g} / \mathrm{ml}$ hygromycin B (Thermo-Fisher). MtTop1 expression was induced in exponentially growing $\left(\mathrm{OD}_{600}=0.5\right)$ cultures of $M$. smegmatis by addition of $50 \mathrm{ng} / \mathrm{ml}$ anhydrotetracycline (ATc; Takara-Clontech) (29). Cell survival was quantified by culturing cells on LB plates containing $200 \mu \mathrm{g} / \mathrm{ml}$ hygromycin B for 3 days at $37^{\circ} \mathrm{C}$.

M. tuberculosis H37Rv (ATCC 25618) strain was grown in 7H9 medium with ADC supplement (HiMedia) as described (28). Up to $2 \times 10^{9}$ Mtb bacilli were incubated in RADAR buffer for $15 \mathrm{~min}$ at $65^{\circ} \mathrm{C}$ with occasional vortexing, washed once in water, plated, and then incubated for four weeks at $37^{\circ} \mathrm{C}$.

\section{RADAR lysis reagent for bacteria}

To perform lysis and RADAR fractionation of bacteria, we used a reagent developed for proteomic analyses of nucleoprotein adducts in human cells (26), with some modifications. The lysis solution for bacteria (LSB) consisted of $5 \mathrm{M}$ guanidinium isothiocyanate (GTC), 1\% Sarkosyl, 1\% 2-mercaptoethanol, $20 \mathrm{mM}$ EDTA, $20 \mathrm{mM}$ Tris$\mathrm{HCl}(\mathrm{pH} 8.0)$ and $0.1 \mathrm{M}$ sodium acetate $(\mathrm{pH} 5.3)$, adjusted to final $\mathrm{pH} 6.5$ with $\mathrm{NaOH}$. The solution was filtered through $0.1 \mu \mathrm{m}$ PES membrane (VWR) and stored in the dark at room temperature. 2-mercaptoethanol was freshly added before use. For alkaline lysis and fractionation, LSB was supplemented with $5 \mathrm{~N} \mathrm{NaOH}$ to the desired concentration.

\section{RADAR/slot blots of Top1-DNA adducts in E. coli}

RADAR fractionation was carried out as previously described $(16,17)$ with some modifications. E. coli cells $\left(\mathrm{OD}_{600}=0.3-0.5\right)$ bearing YpTop1 expression plasmids were cultured in $20 \mathrm{ml}$ of LB broth with or without arabinose inducer and harvested by centrifugation at $3,500 \mathrm{rpm}$ for $10 \mathrm{~min}$. The pellet (approximately $2 \times 10^{10}$ cells) was lysed in $500 \mu \mathrm{LSB}$ supplemented with $0.25 \mathrm{M} \mathrm{NaOH}$, and incubated at $60^{\circ} \mathrm{C}$ for 15 min. Samples were then sonicated with 30 s pulses, 100 amplitude, for 3-4 cycles, and the extract clarified by centrifugation at $21,000 \mathrm{~g}$ for $10 \mathrm{~min}$. To $450 \mu \mathrm{l}$ of resulting supernatant were added $150 \mu \mathrm{l}$ of $8 \mathrm{M} \mathrm{LiCl}$ (final concentration $2 \mathrm{M}$ ) and $600 \mu \mathrm{l}$ isopropanol (equal volume), followed by centrifugation at $21,000 \mathrm{~g}$ for $10 \mathrm{~min}$. The resulting pellet was washed thrice with $75 \%$ ethanol, briefly air dried, resuspended in $400 \mu \mathrm{l}$ freshly prepared $8 \mathrm{mM} \mathrm{NaOH}$, dissolved on the Thermomixer at $2000 \mathrm{rpm}$ at 
135 room temperature and neutralized by addition of $1 \mathrm{M} \mathrm{HEPES}$ free acid solution $(8 \mu \mathrm{l})$.

136 To reduce background of non-covalently bound overexpressed Top1, samples (100 $\mu$ l)

137 were treated with RNase A for $30 \mathrm{~min}$ at $37^{\circ} \mathrm{C}$ (final concentration $20 \mu \mathrm{g} / \mathrm{ml}$ ),

138 supplemented with $350 \mu \mathrm{LS} 1$ and $150 \mu \mathrm{l}$ of $8 \mathrm{M} \mathrm{LiCl}$ solution (final concentration $2 \mathrm{M}$

$139 \mathrm{LiCl}$ ), reprecipitated with equal volume of isopropanol and resuspended in $8 \mathrm{mM} \mathrm{NaOH}$.

140 DNA concentrations were measured with a Qubit assay. Typical recovery was 30-40 $\mu \mathrm{g}$

141 of DNA from $1.8 \times 10^{10}$ E. coli cells. Prior to slot blotting, $100 \mu$ of sample was removed

142 and digested with $0.5 \mu \mathrm{l}$ (12.5 units) Benzonase (Novagen) in the presence of $2 \mathrm{mM}$

$143 \mathrm{MgCl}_{2}$ for $30 \mathrm{~min}$ at $37^{\circ} \mathrm{C}$.

$144 \quad$ For slot blotting, $100 \mu \mathrm{l}$ of sample containing $0.5-1 \mu \mathrm{g}$ of Benzonase-digested

145 DNA in $25 \mathrm{mM}$ sodium phosphate buffer ( $\mathrm{pH}$ 6.5) was applied to a nitrocellulose

146 membrane (Bio-Rad) pre-wet in the same buffer using a vacuum slot blot manifold (Bio-

147 Rad). Membranes were blocked in 0.5\% alkali soluble casein (Novagen) in $10 \mathrm{ml}$ TBST

148 (10 mM Tris-EDTA pH 7.5, 0.1 M NaCl, 0.05\% Tween 20) for $1 \mathrm{hr}$. TRX-tagged YpTop1

149 was detected with polyclonal rabbit anti-thioredoxin antibodies (Abcam, ab26320,

150 1:1,000). Membranes were incubated with primary antibodies for $3 \mathrm{hr}$, washed in TBST

151 and incubated for $1 \mathrm{hr}$ with HRP-conjugated anti-rabbit secondary antibodies (Thermo-

152 Fisher; 1:10,000). All antibodies were diluted in the blocking solution, and all

153 incubations were performed at room temperature. Membranes were developed using

154 Super Signal West Dura (Thermo-Fisher) and imaged on a Bio-Rad Chemidoc XRS

155 Plus Analyzer.

156

157

CsCl density gradient fractionation of YpTop1-DNA adducts in E. coli

$\mathrm{CsCl}$ density gradient fractionation and adduct quantification were based on a protocol developed for bacterial gyrase and topoisomerase IV adducts (30), with some modifications. Briefly, a $50 \mathrm{ml}$ culture $\left(5 \times 10^{10}\right.$ cells) was pelleted, resuspended in $3 \mathrm{ml}$ buffer containing TE (10 mM Tris- $\mathrm{HCl}$ pH 7.5, $1 \mathrm{mM}$ EDTA pH 8.0), 1x protease inhibitor cocktail (Thermo Scientific), and lysozyme (Sigma Aldrich) added to a final concentration of $0.1 \mathrm{mg} / \mathrm{ml}$. Samples were incubated on ice for $10 \mathrm{~min}$, sarkosyl added to final concentration $1 \%$, then following an additional 30 min incubation on ice DNA was sheared by expulsion through $22 \mathrm{G} 1 / 2$ needles followed by centrifugation at 21,000 $\mathrm{g}$ for $10 \mathrm{~min}$. Supernatants $(3 \mathrm{ml})$ were loaded on the top of step gradients preformed in polyallomer tubes (14 by $89 \mathrm{~mm}$, Beckman), containing $2 \mathrm{ml}$ each of 1.82, 1.72, 1.50, and $1.37 \mathrm{~g} / \mathrm{ml} \mathrm{CsCl}$ (Sigma Aldrich) in $10 \mathrm{mM}$ Tris, $1 \mathrm{mM}$ EDTA, pH 8.0. Following centrifugation in a Beckman SW41Ti rotor at $31,000 \mathrm{rpm}$ for $20 \mathrm{hr}$ at $20^{\circ} \mathrm{C}$, the bottom of the tube was punctured, and 14 fractions were collected. DNA was quantified by Qubit assay. To compare levels of adducts formed by WT and mutant YpTop1, equal amounts of DNA from each fraction $(1,000 \mathrm{ng})$ were prepared to load onto the membrane; prior to slot blotting, samples were digested with $0.25 \mu \mathrm{l}$ Benzonase in the 
175 presence of $2 \mathrm{mM} \mathrm{MgCl}_{2}$ for $30 \mathrm{~min}$ at $37^{\circ} \mathrm{C}$. TRX-tagged YpTop1 was detected, as

176 described for YpTop1 RADAR/slot blots.

177

178

179

180

181

182

183

184

185

186

187

188

189

190

191

192

193

194

195

196

197

198

199

200

201

202

203

204

205

206

207

208

209

210

211

212

213

214

\section{RADAR/ELISA assays of YpTop1-DNA adducts in E. coli}

For RADAR fractionation in 96-well microtiter plates, cells $\left(4 \times 10^{7}\right)$ were cultured in $50 \mu l$ medium and protein expression induced with $0.02 \%$ arabinose. Cells were pelleted by centrifugation for $5 \mathrm{~min}$ at 2,700 g, resuspended in $50 \mu \mathrm{l}$ 1x FastBreak ${ }^{\mathrm{TM}}$ Cell Lysis Reagent (Promega) supplemented with RNase A $(20 \mu \mathrm{g} / \mathrm{ml})$ and incubated at $37^{\circ} \mathrm{C}$ for 5 min, followed by addition of $50 \mu \mathrm{LSB}$ premixed with $12 \mathrm{M} \mathrm{LiCl}(1: 6$, final concentration $1 \mathrm{M} \mathrm{LiCl}$ ). After addition of $100 \mu \mathrm{l}$ isopropanol (equal volume) DNA was precipitated by centrifugation for $10 \mathrm{~min}$ at $2,700 \mathrm{~g}$. Pellets were washed with $200 \mu \mathrm{l} 75 \%$ ethanol, briefly air dried and solubilized on the Thermomixer (20 min, 2,000 rpm) in $25 \mu l 8 \mathrm{mM}$ $\mathrm{NaOH}$, followed by neutralization with $1 \mathrm{M}$ HEPES. Typical recovery following culture and fractionation in 96 well plates was $500-700 \mathrm{ng}$ of DNA from $10^{8} \mathrm{E}$. coli cells.

For the homogeneous assay, cells were not pelleted. Instead, $5 \mu$ l0x FastBreak $^{\text {TM }}$ Cell Lysis Reagent supplemented with RNase A was added directly to 50 $\mu \mathrm{l}$ of bacterial culture and incubated on the Thermomixer for $5 \mathrm{~min}$ at $37^{\circ} \mathrm{C}$, then $50 \mu \mathrm{l}$ LSB-LiCl mixture was added and all subsequent steps carried out as above.

Sandwich ELISAs in 96-well format were used to quantify TRX-tagged YpTop1 adducts in samples containing the equivalent of $100 \mathrm{ng}$ DNA per well. Prior to ELISA, DNA was digested with Benzonase in the presence of $2 \mathrm{mM} \mathrm{MgCl}_{2}$. Samples were applied to ELISA plates (Nunc Poly-sorb) pre-coated with rabbit polyclonal anti-TRX capture antibodies (EpiGentek \#A57734, $0.5 \mu \mathrm{g} / \mathrm{ml}$ ). Primary detection of TRX-tagged

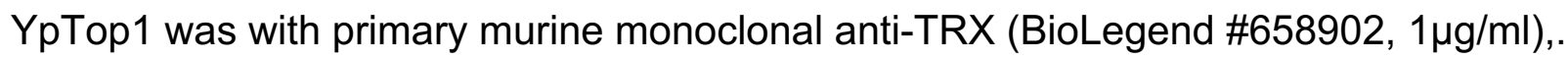
Secondary detection used biotin-conjugated anti-mouse lgG (BioLegend \#405303, 0.5 $\mu \mathrm{g} / \mathrm{ml}$ ), followed by HRP-Streptavidin conjugates (BioLegend \#405210, 1:1,000). All antibody dilutions were in 1x ELISA assay diluent (BioLegend).

$Z^{\prime}$ factors were calculated for assays carried out at different times post-induction (0-5.5 hr) to test the effectiveness of assay conditions and to determine the optimum time at which to assay adducts. Z' was calculated using the formula: $Z^{\prime}=1-\left(3 \sigma_{p}+3 \sigma_{b}\right)$ $\left(\mu_{b}-\mu_{p}\right)$, where $\sigma_{p}$ and $\sigma_{b}$ are the standard deviation of the signals of YpTop1 D117N and YpTop1 WT, respectively; $\mu_{b}$ represents the mean of the signal obtained from YpTop1 WT and $\mu_{p}$ is the mean of YpTop1-D117N.

\section{RADAR/ELISA assays of MtTop1-DNA adducts in M. smegmatis}

After induction of MtTop1 expression with anhydrotetracycline (ATc, Sigma; $50 \mathrm{ng} / \mathrm{ml}$ ), 2 $\mathrm{ml}$ cultures (approximately $2.5 \times 10^{9}$ bacilli) were collected and cells harvested by centrifugation at $21,000 \mathrm{~g}$ for $2 \mathrm{~min}$. Pellets were washed with $1 \mathrm{ml}$ sterile $\mathrm{H}_{2} \mathrm{O}$, 
215 centrifugated as above, and resuspended in $100 \mu \mathrm{l} \mathrm{TE}$. Suspensions were then

216 sonicated for 3-4 cycles of 30 sec pulses, 100 amplitude, and treated with RNase A (10

$217 \mu \mathrm{g} / \mathrm{ml}$ ) for $30 \mathrm{~min}$ at $37^{\circ} \mathrm{C}$. Then, $400 \mu \mathrm{l} \mathrm{LSB}$ was added, followed by $15 \mathrm{~min}$ incubation

218 on the Thermomixer at $60^{\circ} \mathrm{C}$. Extracts were clarified by centrifugation at $21,000 \mathrm{~g}$ for 10

219 min, supernatants $(450 \mu \mathrm{l})$ transferred to new tubes and supplemented with $\mathrm{LiCl}$ (final

220 concentration $2 \mathrm{M} \mathrm{LiCl}$ ). After mixing with equal volume of isopropanol, DNA was

221 precipitated at $21,000 \mathrm{~g}$ for $10 \mathrm{~min}$. DNA pellets were washed with $75 \%$ ethanol, briefly

222 dried in air, and $50 \mu \mathrm{l}$ of $8 \mathrm{mM} \mathrm{NaOH}$ was added prior to solubilization on a

223 Thermomixer followed by neutralization with $1 \mu \mathrm{l} 1 \mathrm{M}$ HEPES. To perform fractionation

224 in 96 deep-well plate format, aliquots of $0.5-2.5 \times 10^{9} \mathrm{M}$. smegmatis cells were processed

225 as above, except all centrifugations were performed at 2,700 g. DNA concentrations

226 were determined by Qubit assay. Prior to ELISA, DNA was digested with Benzonase in

227 the presence of $2 \mathrm{mM} \mathrm{MgCl}_{2}$.

228 For direct ELISA of FLAG-tagged MtTop1 adducts isolated from M. smegmatis,

229 samples were adjusted to 1XELISA coating buffer (BioLegend) and applied to untreated

230 ELISA plates. Samples were absorbed for $2 \mathrm{hr}$ at room temperature or overnight at

$2314^{\circ} \mathrm{C}$. Wells were washed 4 times with $100 \mu \mathrm{l}$ of 1 XPBS, 5 min per wash, then blocked

232 with $100 \mu \mathrm{l}$ of $1 \mathrm{xELISA}$ assay diluent (BioLegend). Following incubation with $1 \mu \mathrm{g} / \mathrm{ml}$

233 mouse monoclonal anti-DDK (FLAG) antibody (60 $\mu \mathrm{l}$ per well, $3 \mathrm{hr}$ at room

234 temperature), plates were washed 4 times for $5 \mathrm{~min}$, then incubated for 45 min with 60

$235 \mu$ l HRP-conjugated secondary goat anti-mouse IgG (Thermo Scientific, 1:5,000) and

236 washed as above. Signal detection was performed using TMB High Sensitivity Solution

237 (BioLegend, \# 421501) according to manufacturer's instructions. Absorbance was read

238 at 450 and $570 \mathrm{~nm}$, and the $A 570$ reading was subtracted as background from the A450

239 signal to correct for background.

\section{$\mathrm{CsCl}$ density gradient fractionation of MtTop1-DNA adducts in $M$. smegmatis} resuspended in $3 \mathrm{ml}$ TE with 1x protease inhibitor cocktail. Samples were sonicated on ice for 4 cycles of $30 \mathrm{Amp}$ and $30 \mathrm{sec}$ pulse time on/off, followed by addition of RNase A (Thermo Fisher) to a final concentration of $10 \mu \mathrm{g} / \mathrm{ml}$ and sarkosyl to a final concentration of $1 \%$, after which samples were incubated for 30 min on ice.

Density gradient centrifugation, fraction collection and DNA quantification were performed as described for YpTop1 adducts in E.coli. Prior to dot blotting, $10 \mu \mathrm{l}$ of each fraction was digested with $0.25 \mu$ Benzonase in the presence of $2 \mathrm{mM} \mathrm{MgCl}_{2}$ for $30 \mathrm{~min}$ at $37^{\circ} \mathrm{C}$. FLAG-tagged MtTop1 was detected with anti-DDK (FLAG) antibodies (Origene, TA50011, 1:2,500). Membranes were incubated with primary antibodies at 
254 anti-mouse secondary antibodies (Thermo-Fisher; 1:10,000). Signal was detected, as

255 described for the RADAR/slot blots of YpTop1-DNA adducts.

\section{RESULTS}

\section{Adducts of cytotoxic YpTop1-D117N accumulate upon expression in E. coli}

We assayed adducts formed by YpTop1 WT and YpTop1-D117N bearing N-terminal thioredoxin (TRX) tags, expressed in E. coli. YpTop1-D117N bears a mutation in the TOPRIM domain (Fig. 1B) that eliminates a negatively charged residue required for $\mathrm{Mg}^{2+}$ interaction, rendering the protein defective in DNA religation and extremely cytotoxic upon expression in E. coli cells $(11,12)$. YpTop1 WT and YpTop1-D117N were expressed from an arabinose-inducible promoter, and $\mathrm{OD}_{600}$ of the cultures determined during $4 \mathrm{hr}$ following induction of protein expression by addition of arabinose. Induction did not affect viability of cells expressing YpTop1 WT, but a clear drop in $\mathrm{OD}_{600}$ occurred in cells expressing YpTop1-D117N (Fig. 2A). Plating assays carried out at $3 \mathrm{hr}$ postinduction confirmed that expression of YpTop1-D117N, but not YpTop1 WT diminished cell viability (Fig. 2B). Analysis of DNA adducts by RADAR/slot blot identified only a faint signal in samples from uninduced cultures or from induced cultures expressing YpTop1 WT, but an intense signal in samples from induced cultures expressing YpTop1-D117N (Fig. 3C). Thus, impaired survival caused by induction of YpTop-D117N expression in E. coli correlated with accumulation of DNA adducts as assayed by RADAR/slot blot.

We used the classical approach of $\mathrm{CsCl}$ density gradient fractionation (26) to confirm the results of RADAR/slot blot. Extracts of cultures expressing TRX-tagged YpTop1 WT or YpTop1-D117N, uninduced or induced by 150 min culture with arabinose, were fractionated on a $\mathrm{CsCl}$ step gradient, and the DNA concentration of each fraction was measured and plotted, showing that DNA peaked in fractions 4-6 (Fig. 2D). Slot blots showed that signals from free protein were evident in fractions 7-8 of all samples, which are near the top of the gradient (Fig. 2E). The free protein signal increased in response to induction of Top1 expression with arabinose, as expected. The DNA peak-containing fractions of intermediate density (fractions 4-6) exhibited clear signals only in arabinose-induced cultures, with a stronger signal evident in the fractions from cells expressing YpTop1-D117N (Fig. 2E, right). To assess adduct recovery from induced cells, the Top1 signal was quantified by densitometry (Fig. 2F). This showed that more adducts accumulated in E. coli cells expressing YpTop1-D117N mutant than YpTop1 WT, validating the results of the RADAR/slot blot. 
294 To further streamline RADAR quantification of adducts, we scaled down cell numbers to

295 enable adduct quantification in microtiter plates by RADAR/ELISA assay (17). As

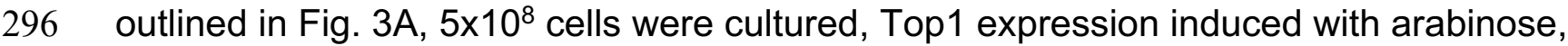

297 pelleted, lysed by treatment with chaotropic salts and detergent, and the sample then

298 alcohol precipitated to recover nucleic acids and covalently bound proteins,

299 resuspended, and finally aliquoted to quantify DNA recovery by Qubit assay and detect

300 the Top1 signal by ELISA. The microtiter plate format allows multiple samples to be

301 processed in parallel, facilitating kinetic analysis of adduct accumulation.

302 RADAR/ELISA assays of adducts during the first $5.5 \mathrm{hr}$ after arabinose induction of

303 YpTop1 expression showed that the signal increased through $2 \mathrm{hr}$ and dropped

304 dramatically at $4 \mathrm{hr}$ (Fig. 3B). This timing correlated with the reduction of $\mathrm{OD}_{600}$ in the culture expressing YpTop1-D117N (Fig. 2B), suggesting that adducts might be released into the medium upon cell lysis.

To assay adducts in both intact cells and the culture medium, we devised a "homogenous assay" in which cells were not pelleted prior to addition of lysis buffer. Instead, cell lysis and ethanol precipitation were carried out on the entire contents of each well of a 96-well micro-titer plate. RADAR/ELISA assay showed that the protein signal as detected by this approach peaked at $2 \mathrm{hr}$ and then decreased gradually (Fig. $3 \mathrm{C})$, evidence of reduced sensitivity of the assay to cell lysis.

To establish whether the homogeneous RADAR/ELISA assay was sufficiently quantitative and reproducible for high throughput applications, we determined Z' factors for different time points of Top1 induction. Assays with Z'>0.5 are considered excellent, and this criterion was satisfied by homogeneous RADAR/ELISA assay of Top1-DNA adducts at 1-2 hr post-induction (Fig. 3D).

RADAR lysis conditions efficiently recover DNA from Mycobacterium sp. To extend the RADAR/ELISA assay to a context other than E. coli, we focused on Mycobacteria. Mycobacterium tuberculosis (Mtb) causes tuberculosis (TB), a major challenge to human health worldwide and the deadliest infectious disease, after AIDS (WHO, 2019; https://www.who.int/tb/en/). Mtb encodes a single type IA DNA topoisomerase (MtTop1) that is crucial for viability and predicted to be a drug target (3133). Mycobacteria are notoriously difficult to disrupt, as the cells are protected by a tough outer layer composed of lipids, mycolic acids, polysaccharides (arabinoglycan) and peptidoglycans that make them highly resistant to lysis by standard chemical or enzymatic approaches. Strikingly, treatment with LSB at $65^{\circ} \mathrm{C}$ was toxic to the virulent Mtb H37Rv (ATCC 25618) strain, which would enable treated Mtb cells to be handled outside a BSL-3 facility. Mtb are very slow-growing, so for assay development we turned instead to $M$. smegmatis and BCG. We found that we were conveniently able to achieve DNA yields on the order of $20 \%$ from both bacteria by incubation of cells at $65^{\circ} \mathrm{C}$ for $10 \mathrm{~min}$ in RADAR LSB, followed by isopropanol precipitation and centrifugation 
334 at 21,000 $\mathrm{g}$ for $10 \mathrm{~min}$ using a standard benchtop microfuge (Fig. 4A). DNA yields from

335 M. smegmatis or BCG were considerably improved by sonication, which is useful for

336 large-scale preparation but requires specialized equipment for analysis of small

337 volumes in microplate format.

RADAR/ELISA detects MtTop1 DNA adducts in $M$. smegmatis cells

To analyze adduct formation by MtTop1 expressed in $M$. smegmatis, we modified a construct for tetracycline-inducible expression of MtTop1 to carry an N-terminal FLAG (DDK) tag for immunodetection. We then generated mutations in MtTop1 at two positions reported to cause toxicity in other bacterial Top1 proteins (Fig. 4B, above). These included a D111N mutation, corresponding to the YpTop1-D117N mutation toxic in E. coli (11); and a G116S mutation, corresponding to mutations in Yp or EcTop1 that caused a dramatic decrease in cell viability and induced an SOS response in $E$. coli

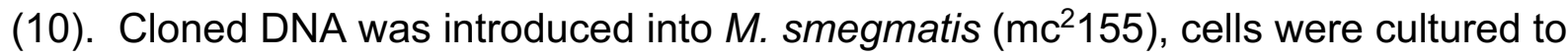
early log phase $\left(\mathrm{OD}_{600} \sim 0.5\right)$, and MtTop1 expression induced by addition of anhydrotetracycline (ATc, $50 \mathrm{ng} / \mathrm{ml}$ ). Analysis of viable CFU in cells plated at $8 \mathrm{hr}$ postinduction found that expression of MtTop1-D111N or MtTop1-G116S reduced viability only 2-fold, as shown by analysis of CFU recovered in two independent experiments (Fig. 4B, below). The limited toxicity may reflect structural differences between MtTop1 and EcTop1 (9) and/or physiological differences between E. coli and M. smegmatis.

To quantify adducts by RADAR/ELISA assay, cells expressing MtTop1 WT, $\mathrm{D} 111 \mathrm{~N}$ or $\mathrm{G} 116 \mathrm{~S}$ were cultured to $\mathrm{OD}=0.5$, then ATc added and cells cultured 0, 2 or 4 hr post-induction. Adducts were then enriched by RADAR fractionation, and MtTop1DNA complexes detected by direct ELISA with antibody to the N-terminal FLAG tag (Fig. 4C). Comparable ELISA signals were evident in RADAR fractions of cells expressing WT and mutant enzymes prior to induction of MtTop1 expression $(t=0)$. The signal from extracts of cells expressing MtTop1 WT or the G116S mutant increased through the $4 \mathrm{hr}$ induction period, and the signal from extracts of cells expressing the $\mathrm{D} 111 \mathrm{~N}$ mutant increased at $2 \mathrm{hr}$ then dropped slightly by $4 \mathrm{hr}$.

We carried out $\mathrm{CsCl}$ buoyant density fractionation to confirm results of RADAR/ELISA. M. smegmatis cells expressing WT or mutant MtTop1 were cultured for $4 \mathrm{hr}$ with $50 \mathrm{ng} / \mathrm{ml} \mathrm{ATc}$, pelleted, lysed by sonication, and samples resolved on $\mathrm{CsCl}$ step gradients. Fractions were collected by puncturing the bottom of each tube. Fractions 5-9 were shown to contain the peak of DNA in each sample (Fig. 4D). Proteins in aliquots of each fraction were captured on a slot blot and FLAG-tagged Top1 detected with anti-FLAG tag antibodies (Fig. 4E). Adduct recovery was quantified by summing signals determined by densitometry of DNA-containing fractions (5-9) and normalizing to DNA concentration (Fig. 4F). FLAG-Top1 signals at $4 \mathrm{hr}$ post-induction 
373 determined by density gradient centrifugation were found to be comparable to those

374 determined by RADAR/ELISA.

\section{DISCUSSION}

Here we report a straightforward assay that directly quantifies bacterial Top1-DNA adducts by ELISA assay of RADAR-fractionated cells. We demonstrate that RADAR/ELISA assay can be used to quantify adducts formed by Top1 encoded by two different pathogenic bacteria, Y. pestis and Mtb.

Analysis of YpTop1 expressed in E. coli showed that RADAR/ELISA assay offers a simple approach for characterization of Top1 mutants YpTop1 WT and its highly toxic mutant derivative YpTop1-D117N were expressed in E. coli. Adducts formed by the YpTop1-D117N mutant were detected at greater levels than YpTop1 WT adducts, as assayed by RADAR/slot blot, RADAR/ELISA, or $\mathrm{CsCl}$ fractionation. The increased level of adducts suggests that this mutant, which is deficient in religation, mimics the effect of treatment with topoisomerase poisons.

The results presented here report analysis of $\mathrm{N}$-terminal tagged recombinant Top1 expressed from inducible promoters and detected with epitope-specific tags. The tag enables assays of adducts formed by topoisomerases for which suitable, specific antibodies are not available commercially. The essay is optimized for bacterial Top1, but it can be extended to other epitope-tagged topoisomerase targets, such as gyrases. The utility of a tag will be limited if proteolytic cleavage occurs at sites between the tag and the Top1-DNA covalent bond. We have recently shown that proteolytic repair of human topoisomerase 1 can be assessed by RADAR fractionation combined with mass spectrometry (15). That approach could be readily adopted to determine whether bacterial topoisomerase-DNA adducts were targets of proteolytic cleavage.

Cell lysis may accompany cell killing that results from drug treatment. We found that fractionation of adducts from both cells and culture medium offers a work-around, although even with this approach adduct detection diminished at later times, likely reflecting proteolysis. This highlights the importance of kinetic analysis in optimizing assay conditions. RADAR/ELISA simplifies kinetic analyses by enabling large numbers of samples to be assayed in parallel.

RADAR/ELISA proved able to detect Top1-DNA adducts fractionated from either E. coli or M. smegmatis cells, providing a foundation for extension of the assay to other bacteria. The tough cell wall of Mycobacteria presents a considerable challenge to many biochemical approaches, but sufficient DNA was recovered by RADAR fractionation to enable quantification of adducts. This suggests that the approach can be extended to other bacteria. The results reported here thus provide a general foundation for discovery and optimization of drugs that poison bacterial Top1 using standard high-throughput approaches. 
SUPPLEMENTARY DATA - none.

\section{ACKNOWLEDGEMENTS}

417 We thank Dr. Yuk-Ching Tse-Dinh for providing E. coli strains bearing YpTop1 and for valuable advice on experimental design.

\section{FUNDING}

421 National Institute of Allergy and Infectious Disease of the U.S. National Isntitutes of 422 Health award number R21 Al123501 to N.M.

\section{CONFLICT OF INTEREST - none.}

\section{REFERENCES}

1. Tse-Dinh, YC. 2015. Targeting bacterial topoisomerase I to meet the challenge of finding new antibiotics. Future Med Chem 7: 459-471. https://www.ncbi.nlm.nih.gov/pubmed/25875873

2. Tse-Dinh, YC. 2016. Targeting bacterial topoisomerases: how to counter mechanisms of resistance. Future Med Chem 8: 1085-1100.

https://www.ncbi.nlm.nih.gov/pubmed/27285067

3. Chen, SH, Chan, NL, Hsieh, TS. 2013. New mechanistic and functional insights into DNA topoisomerases. Annu Rev Biochem 82: 139-170.

https://www.ncbi.nlm.nih.gov/pubmed/23495937

4. Aldred, KJ, Kerns, RJ, Osheroff, N. 2014. Mechanism of quinolone action and resistance. Biochemistry 53: 1565-1574. http://www.ncbi.nlm.nih.gov/entrez/query.fcgi?cmd=Retrieve\&db=PubMed\&dopt= Citation\&list uids $=24576155$

5. Pendleton, M, Lindsey, RH, Jr., Felix, CA, Grimwade, D, Osheroff, N. 2014. Topoisomerase II and leukemia. Ann N Y Acad Sci 1310: 98-110. https://www.ncbi.nlm.nih.gov/pubmed/24495080

6. Pommier, Y, Leo, E, Zhang, H, Marchand, C. 2010. DNA topoisomerases and their poisoning by anticancer and antibacterial drugs. Chem Biol 17: 421-433. https://www.ncbi.nlm.nih.gov/pubmed/20534341

\section{Nagaraja, V, Godbole, AA, Henderson, SR, Maxwell, A. 2017. DNA} topoisomerase I and DNA gyrase as targets for TB therapy. Drug Discov Today 22: 510-518. https://www.ncbi.nlm.nih.gov/pubmed/27856347

8. Zhu, CX, Tse-Dinh, YC. 2000. The acidic triad conserved in type IA DNA topoisomerases is required for binding of $\mathrm{Mg}(\mathrm{II})$ and subsequent conformational 
change. J Biol Chem 275: 5318-5322.

https://www.ncbi.nlm.nih.gov/pubmed/10681504

9. Cao, N, Tan, K, Annamalai, T, Joachimiak, A, Tse-Dinh, YC. 2018. Investigating mycobacterial topoisomerase I mechanism from the analysis of metal and DNA substrate interactions at the active site. Nucleic Acids Res 46: 7296-7308. https://www.ncbi.nlm.nih.gov/pubmed/29905859

10. Cheng, B, Shukla, S, Vasunilashorn, S, Mukhopadhyay, S, Tse-Dinh, YC. 2005. Bacterial cell killing mediated by topoisomerase I DNA cleavage activity. J Biol Chem 280: 38489-38495. http://www.ncbi.nlm.nih.gov/entrez/query.fcgi?cmd=Retrieve\&db=PubMed\&dopt= Citation\&list uids $=16159875$

11. Cheng, B, Annamalai, T, Sorokin, E, Abrenica, M, Aedo, S, Tse-Dinh, YC. 2009. Asp-to-Asn substitution at the first position of the DxD TOPRIM motif of recombinant bacterial topoisomerase I is extremely lethal to $\mathrm{E}$. coli. J Mol Biol 385: 558-567.

http://www.ncbi.nlm.nih.gov/entrez/query.fcgi?cmd=Retrieve\&db=PubMed\&dopt= Citation\&list uids $=19013470$

12. Bhat, AG, Leelaram, MN, Hegde, SM, Nagaraja, V. 2009. Deciphering the distinct role for the metal coordination motif in the catalytic activity of Mycobacterium smegmatis topoisomerase I. J Mol Biol 393: 788-802. https://www.ncbi.nlm.nih.gov/pubmed/19733176

13. Narula, G, Becker, J, Cheng, B, Dani, N, Abrenica, MV, Tse-Dinh, YC. 2010. The DNA relaxation activity and covalent complex accumulation of Mycobacterium tuberculosis topoisomerase I can be assayed in Escherichia coli: application for identification of potential FRET-dye labeling sites. BMC Biochem 11: 41. https://www.ncbi.nlm.nih.gov/pubmed/20920291

14. Rani, P, Nagaraja, V. 2019. Genome-wide mapping of Topoisomerase I activity sites reveal its role in chromosome segregation. Nucleic Acids Res 47: 14161427. https://www.ncbi.nlm.nih.gov/pubmed/30566665

15. Kiianitsa, K, Maizels, N. 2020. The "adductome": a limited repertoire of adducted proteins in human cells. DNA Repair.

https://doi.org/10.1016/i.dnarep.2020.102825

16. Kiianitsa, K, Maizels, N. 2013. A rapid and sensitive assay for DNA-protein covalent complexes in living cells. Nucleic Acids Res 41: e104. http://www.ncbi.nlm.nih.gov/entrez/query.fcgi?cmd=Retrieve\&db=PubMed\&dopt= Citation\&list uids $=23519618$

17. Kiianitsa, K, Maizels, N. 2014. Ultrasensitive isolation, identification and quantification of DNA-protein adducts by ELISA-based RADAR assay. Nucleic Acids Res 42: e108. http://www.ncbi.nlm.nih.gov/pubmed/24914050 
18. Aldred, KJ, Schwanz, HA, Li, G, Williamson, BH, McPherson, SA, Turnbough, CL, Jr., Kerns, RJ, Osheroff, N. 2015. Activity of quinolone CP-115,955 against bacterial and human type II topoisomerases is mediated by different interactions. Biochemistry 54: 1278-1286. https://www.ncbi.nlm.nih.gov/pubmed/25586498

19. Aparicio, T, Baer, R, Gottesman, M, Gautier, J. 2016. MRN, CtIP, and BRCA1 mediate repair of topoisomerase II-DNA adducts. J Cell Biol 212: 399-408. https://www.ncbi.nlm.nih.gov/pubmed/26880199

20. Velichko, AK, Petrova, NV, Razin, SV, Kantidze, OL. 2015. Mechanism of heat stress-induced cellular senescence elucidates the exclusive vulnerability of early S-phase cells to mild genotoxic stress. Nucleic Acids Res 43: 6309-6320. https://www.ncbi.nlm.nih.gov/pubmed/26032771

21. Vaz, B, Popovic, M, Newman, JA, Fielden, J, Aitkenhead, H, Halder, S, Singh, AN, Vendrell, I, Fischer, R, Torrecilla, I et al. 2016. Metalloprotease SPRTN/DVC1 Orchestrates Replication-Coupled DNA-Protein Crosslink Repair. Mol Cell 64: 704-719. https://www.ncbi.nlm.nih.gov/pubmed/27871366

22. Aldred, KJ, Payne, A, Voegerl, O. 2019. A RADAR-Based Assay to Isolate Covalent DNA Complexes in Bacteria. Antibiotics (Basel) 8. https://www.ncbi.nlm.nih.gov/pubmed/30818799

23. Mohni, KN, Wessel, SR, Zhao, R, Wojciechowski, AC, Luzwick, JW, Layden, H, Eichman, BF, Thompson, PS, Mehta, KPM, Cortez, D. 2019. HMCES Maintains Genome Integrity by Shielding Abasic Sites in Single-Strand DNA. Cell 176: 144153 e113. https://www.ncbi.nlm.nih.gov/pubmed/30554877

24. Quinones, JL, Thapar, U, Yu, K, Fang, Q, Sobol, RW, Demple, B. 2015. Enzyme mechanism-based, oxidative DNA-protein cross-links formed with DNA polymerase beta in vivo. Proc Natl Acad Sci U S A 112: 8602-8607. https://www.ncbi.nlm.nih.gov/pubmed/26124145

25. Hu, Q, Klages-Mundt, N, Wang, R, Lynn, E, Kuma Saha, L, Zhang, H, Srivastava, M, Shen, X, Tian, Y, Kim, H et al. 2020. The ARK Assay Is a Sensitive and Versatile Method for the Global Detection of DNA-Protein Crosslinks. Cell Rep 30: 1235-1245 e1234. https://www.ncbi.nlm.nih.gov/pubmed/31995761

26. Nitiss, JL, Soans, E, Rogojina, A, Seth, A, Mishina, M. 2012. Topoisomerase assays. Curr Protoc Pharmacol Chapter 3: Unit 33. https://www.ncbi.nlm.nih.gov/pubmed/22684721

27. Minch, KJ, Rustad, TR, Peterson, EJ, Winkler, J, Reiss, DJ, Ma, S, Hickey, M, Brabant, W, Morrison, B, Turkarslan, S et al. 2015. The DNA-binding network of Mycobacterium tuberculosis. Nat Commun 6: 5829. https://www.ncbi.nlm.nih.gov/pubmed/25581030

28. Mdluli, K, Sherman, DR, Hickey, MJ, Kreiswirth, BN, Morris, S, Stover, CK, Barry, CE, 3rd. 1996. Biochemical and genetic data suggest that InhA is not the primary 
target for activated isoniazid in Mycobacterium tuberculosis. J Infect Dis 174: 1085-1090. https://www.ncbi.nlm.nih.gov/pubmed/8896513

29. Ehrt, S, Guo, XV, Hickey, CM, Ryou, M, Monteleone, M, Riley, LW, Schnappinger, D. 2005. Controlling gene expression in mycobacteria with anhydrotetracycline and Tet repressor. Nucleic Acids Res 33: e21. https://www.ncbi.nlm.nih.gov/pubmed/15687379

30. Aedo, S, Tse-Dinh, YC. 2012. Isolation and quantitation of topoisomerase complexes accumulated on Escherichia coli chromosomal DNA. Antimicrob Agents Chemother 56: 5458-5464. https://www.ncbi.nlm.nih.gov/pubmed/22869559

31. Ahmed, W, Menon, S, Godbole, AA, Karthik, PV, Nagaraja, V. 2014. Conditional silencing of topoisomerase I gene of Mycobacterium tuberculosis validates its essentiality for cell survival. FEMS Microbiol Lett 353: 116-123. https://www.ncbi.nlm.nih.gov/pubmed/24593153

32. Ravishankar, S, Ambady, A, Awasthy, D, Mudugal, NV, Menasinakai, S, Jatheendranath, S, Guptha, S, Sharma, S, Balakrishnan, G, Nandishaiah, R et al. 2015. Genetic and chemical validation identifies Mycobacterium tuberculosis topoisomerase I as an attractive anti-tubercular target. Tuberculosis (Edinb) 95: 589-598. https://www.ncbi.nlm.nih.gov/pubmed/26073894

33. Ahmed, W, Menon, S, Karthik, PV, Nagaraja, V. 2015. Reduction in DNA topoisomerase I level affects growth, phenotype and nucleoid architecture of Mycobacterium smegmatis. Microbiology 161: 341-353.

https://www.ncbi.nlm.nih.gov/pubmed/25516959 
FIGURE LEGENDS

Figure 1. Topoisomerase 1 forms DNA adducts.

(A) Top line: Bacterial topoisomerase 1 (Top1) nicks a single strand of the backbone of supercoiled DNA to form a covalent 5'-phosphoryl-tyrosine linkage and relax the supercoil. The adduct is released upon DNA religation, which may be accompanied by restored supercoiling, as shown. Drugs (middle line) or mutations (bottom line) that prevent religation ("topoisomerase poisons") impair release of the protein-DNA adduct to cause cell killing. Images of supercoiled and relaxed circles from Wikicommons. (B) Conserved Top1 TOPRIM domain sequences from Y. pestis (Yp), E. coli (Ec), $M$. tuberculosis (Mt) and M. smegmatis (Ms).

(C) Cell lysis in RADAR buffer (chaotropic salts and detergent) followed by alcohol precipitation enriches protein-DNA protein adducts, which are then captured for immunodetection by ELISA assay (shown) or slot blot.

Figure 2. Quantification of YpTop1 WT and YpTop1-D117N DNA adducts by RADAR slot blot and $\mathrm{CsCl}$ buoyant density fractionation

(A) Growth kinetics $\left(\mathrm{OD}_{600}\right)$ of cultures of $E$. coli bearing arabinose-inducible expression clones for TRX-tagged YpTop1 WT and YpTop1-D117N, assayed from 0-240 minutes after induction with indicated concentration of arabinose.

(B) Representative assay of CFU recovered from E. coli expressing YpTop1 WT and YpTop1-D117N, assayed at 180 minutes of culture with $0.02 \%$ arabinose. (C) Slot blot of RADAR-fractionated extracts isolated from E. coli at 150 min postinduction of expression of YpTop1 WT or YpTop1-D117N by culture with arabinose. Immunodetection was performed with antibodies to TRX epitope. (D) Quantification of DNA recovery by $\mathrm{CsCl}$ gradient fractionation of extracts of cells that were uninduced or induced by 150 min culture with arabinose.

(E) Slot blot of extracts of uninduced and induced cells fractionated by $\mathrm{CsCl}$ density gradient centrifugation. Bottom, bottom of gradient.

(F) Quantification of TRX-tagged YpTop1 derivatives recovered by $\mathrm{CsCl}$ gradient fractionation and detected by slot blot.

Figure 3. Reproducible RADAR/ELISA assay in microtiter format.

(A) Flowchart for adduct recovery from cells cultured in microtiter format. $\mathrm{D} 117 \mathrm{~N}$ at indicated times after induction of expression with arabinose $(0.2 \%)$. 
594 (D) Z' factors of homogenous Top1 RADAR/ELISA assays calculated at indicated times 595 post-induction of protein expression.

597 Figure 4. RADAR/ELISA quantifies MtTop1 DNA adducts in $M$. smegmatis

598 (A) Recovery of DNA from (left) M. smegmatis (ng/108 cells) or (right) BCG. Bacterial

599 lysis was performed in LSB supplemented with $\mathrm{NaOH}$ at indicated concentrations and in

600 the absence/presence 2\% NP-40, followed by alcohol precipitation to recover DNA. Kit:

601 DNA was isolated using the commercial MagJET Genomic DNA Kit.

602 (B) Above, TOPRIM domain sequences of WT and mutant MtTop1. Below,

603 CFU recovered from $M$. smegmatis expressing MtTop1 WT and indicated mutants at 8

$604 \mathrm{hr}$ after induction of MtTop1 expression with ATc. Colonies were counted after 3 days

605 growth at $37^{\circ} \mathrm{C}$ on plates containing $200 \mu \mathrm{g} / \mathrm{ml}$ hygromycin B. Shown are absolute

606 values and survival normalized to cells expressing MtTop1 WT.

607 (C) RADAR/ELISA assay of FLAG-MtTop1 signal from pelleted cells expressing WT

608 and mutant FLAG-MtTop1 at indicated times after induction of expression with ATc (50

$609 \mathrm{ng} / \mathrm{ml})$.

610 (D) Quantification of DNA recovered in $\mathrm{CsCl}$ gradient fractions of extracts of $M$.

611 smegmatis cells expressing FLAG-MtTop1 WT and indicated mutants following $4 \mathrm{hr}$

612 induction with ATc. Bottom, bottom of gradient.

613 (E) Slot blot detection of FLAG-tagged MtTop1 derivatives in indicated CsCl density

614 gradient fractions. Bottom, bottom of gradient.

615 (F) Quantification of recovery of FLAG-MtTop1 from WT and mutant derivatives. Each

616 signal represents the total from fractions 5-9, which contain the peak of DNA. 
A
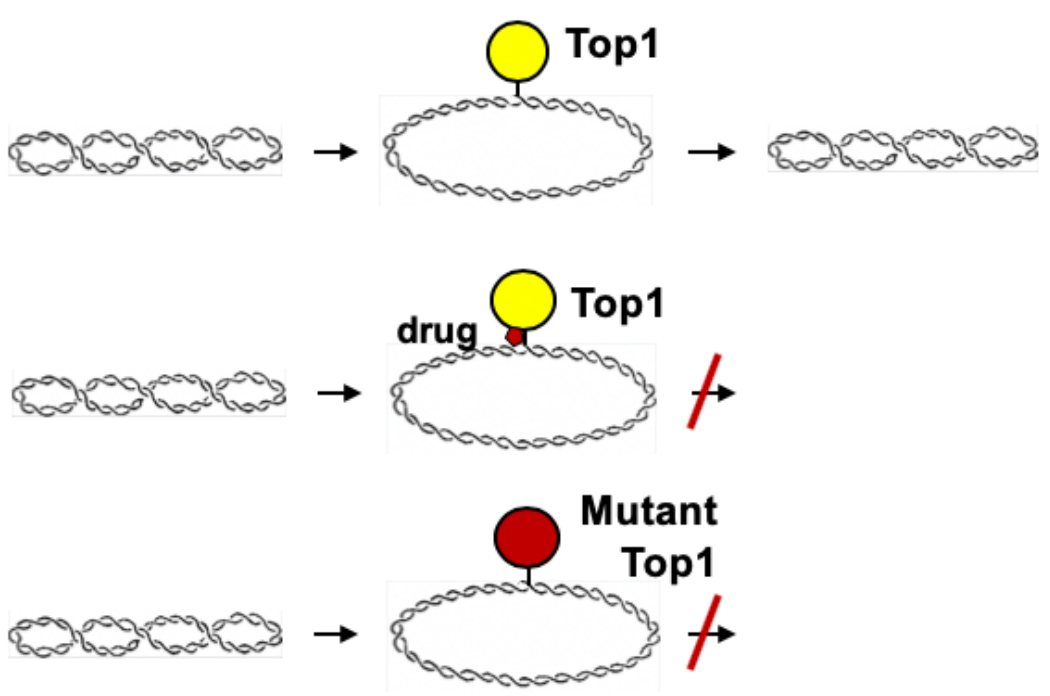

B

\section{TOP1 TOPRIM Domain}

$\begin{array}{lll}\text { Yp } & 117 & \text { DLDREG } \\ \text { EC } & 107 & \text {------ } \\ \text { Mt } & 111 & \text {-G---- } \\ \text { Ms } & 108 & \text {-G---- }\end{array}$

C

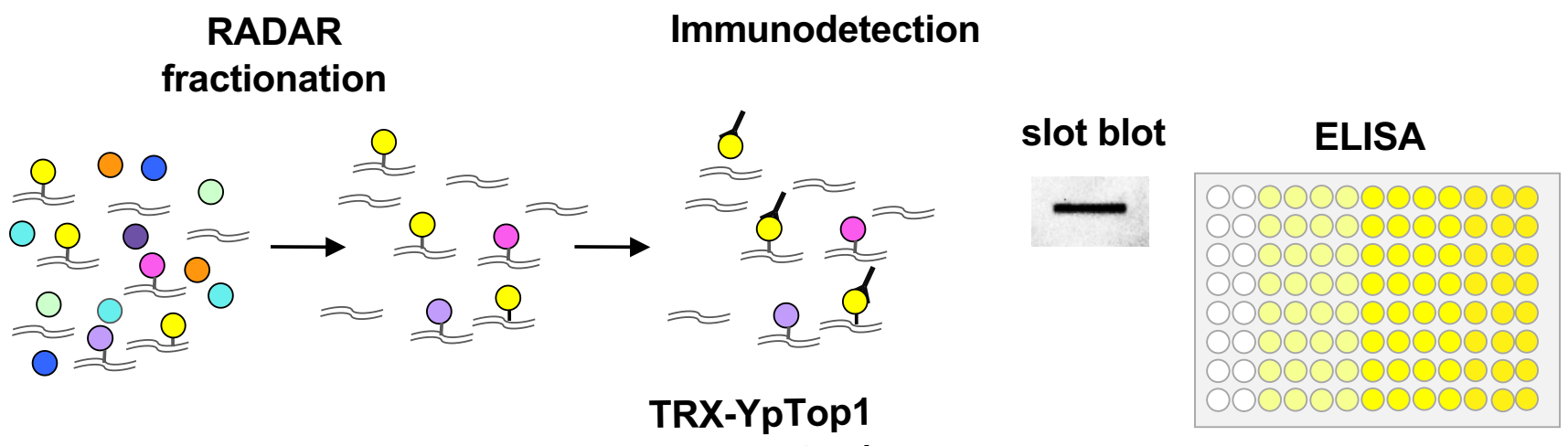
FLAG-MtTop1 


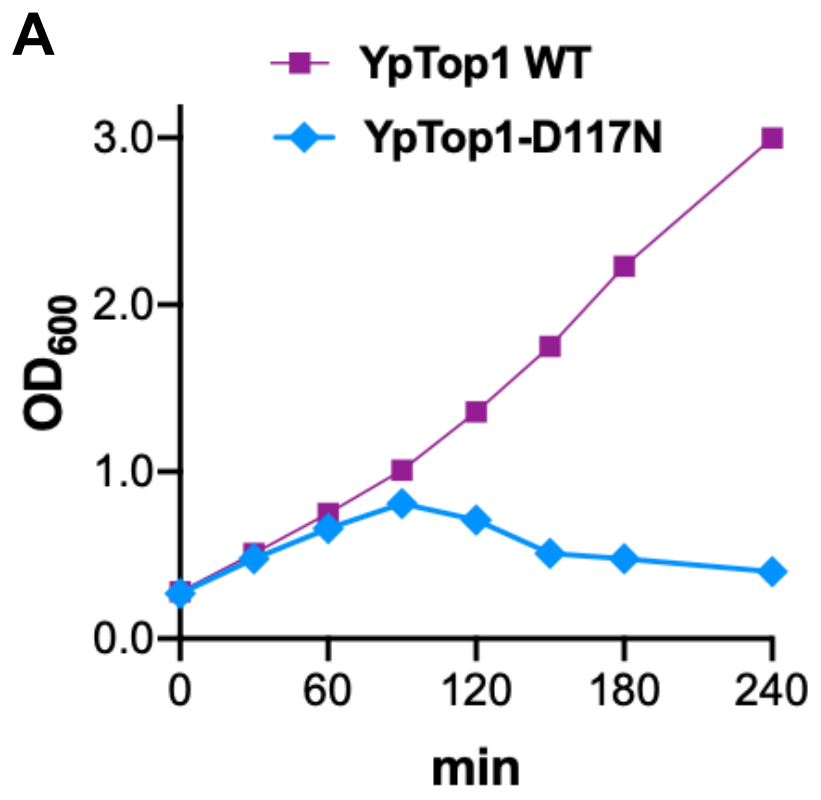

D

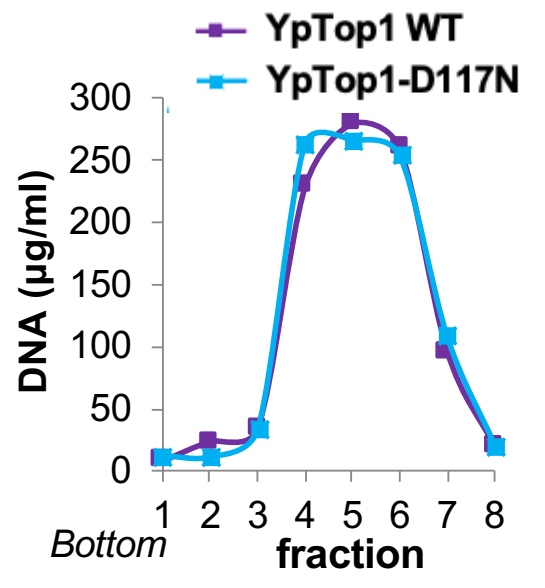

B

\begin{tabular}{ccc}
\hline \multicolumn{3}{c}{ E. coli CFU $(\mathbf{x 1 0 - 6 )}$ at $\mathbf{3 ~ h r}$} \\
\hline Ara & $\begin{array}{c}\text { YpTop1 } \\
\text { WT }\end{array}$ & $\begin{array}{c}\text { YpTop1- } \\
\text { D117N }\end{array}$ \\
0 & 1244 & 1240 \\
$0.02 \%$ & 1189 & 0 \\
\hline
\end{tabular}

C

$\mathbf{F}$

TRX-YpTop 1

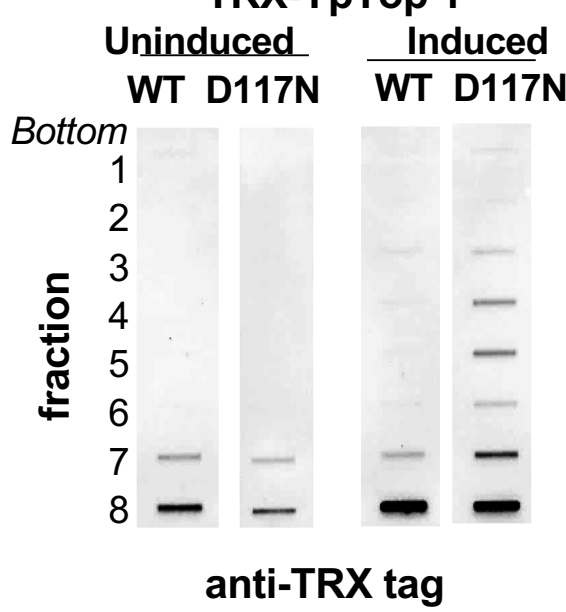

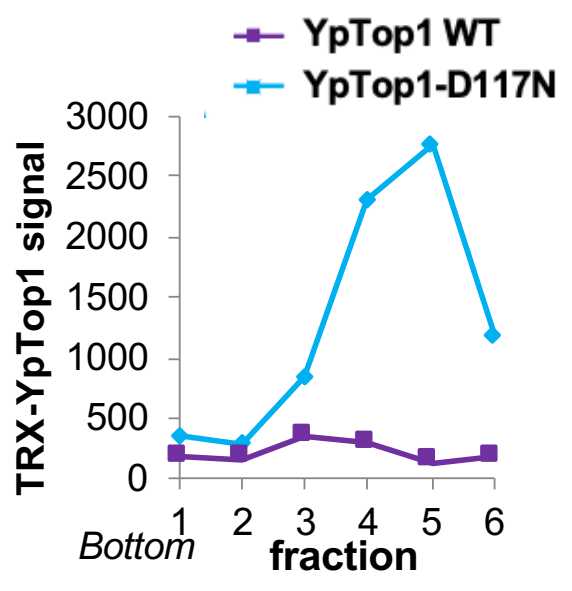


A

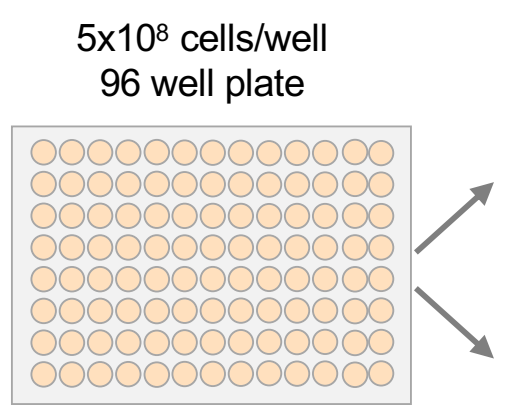

\section{Assay Cell Pellets}

1. Pellet cells

2. Add LSB

3. Precipitate with alcohol

Assay Cells+Medium

1. Add LSB to cell cultures

2. Precipitate with alcohol

B

Assay cell pellets

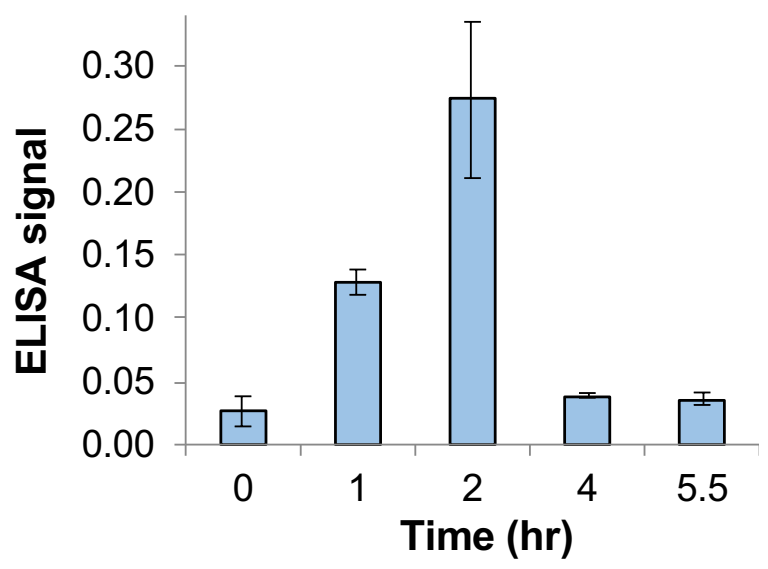

C

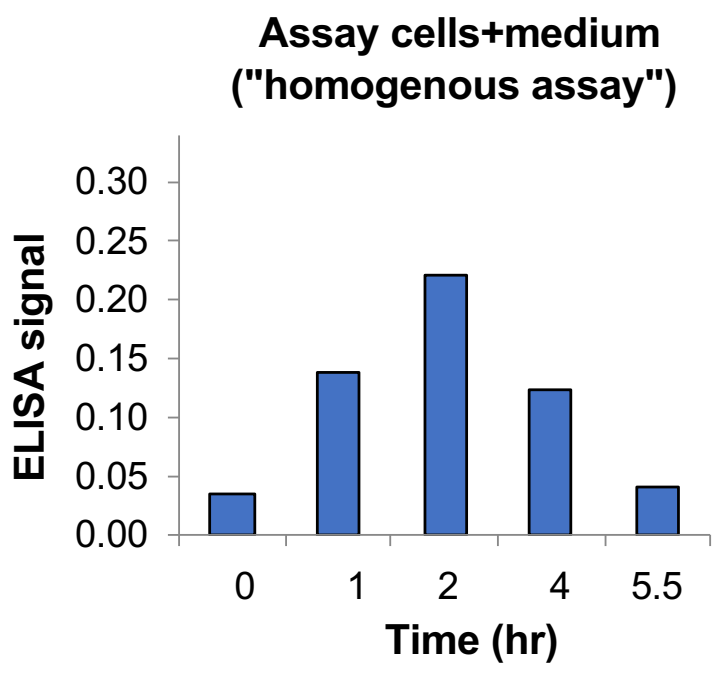

D

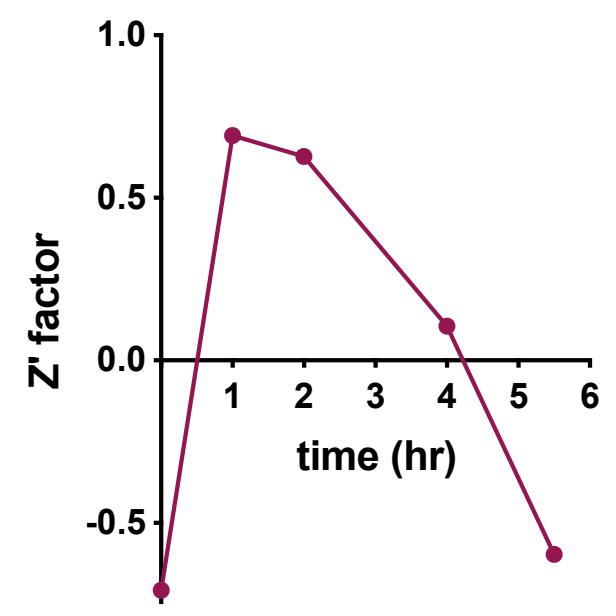


A
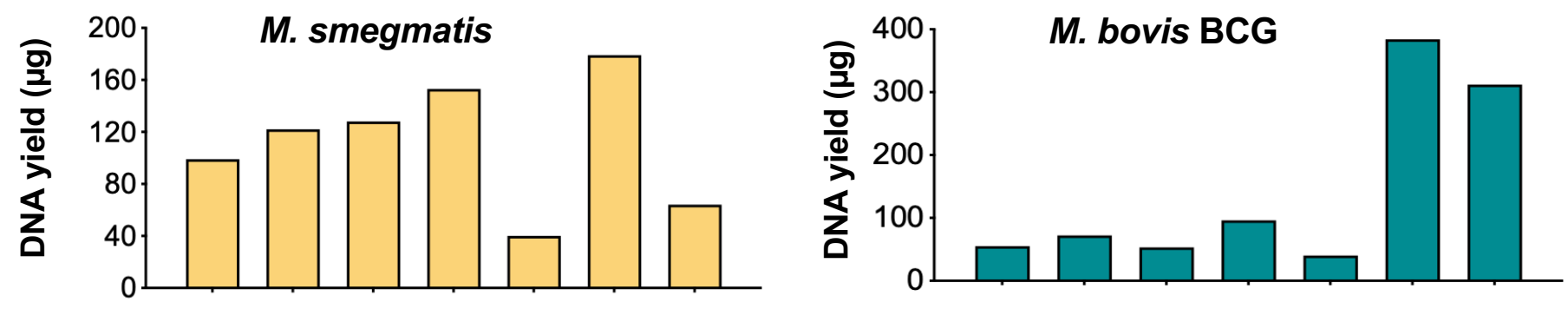

$\mathrm{NaOH}: \quad 0.12 \quad 0.12 \quad 0.25 \quad 0.5 \quad 0.25 \quad 0.25 \quad$ kit

${ }^{\circ} \mathrm{C}: \quad 65^{\circ} \quad 65^{\circ} \quad 65^{\circ} \quad 65^{\circ} \quad 25^{\circ} \quad 25^{\circ}$

Sonication:

NP40: - $\quad$ - $\quad+\quad-\quad$ -

B MtTop1 TOPRIM domain

residues 111-116)

WT DGDREG

D111N N-----

G116S -----S

M. smegmatis CFU $\left(\times 10^{-6}\right)$ at $8 \mathrm{hr}$

\begin{tabular}{ccc}
\hline & CFU & normalized \\
\hline MtTop1 WT & $1097 \pm 42$ & 1 \\
MtTop1-D111N & $590 \pm 36$ & 0.55 \\
\hline MtTop1-G116S & $656 \pm 54$ & 0.57 \\
\hline
\end{tabular}

D

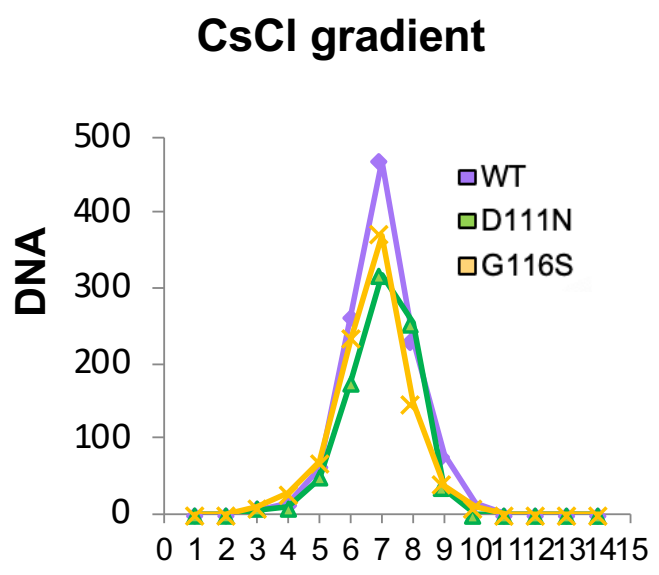

Bottom fraction

E

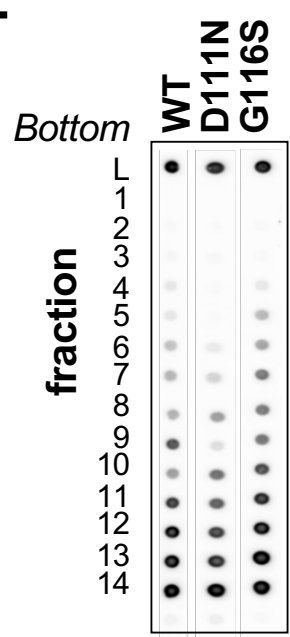

$\mathbf{F}$

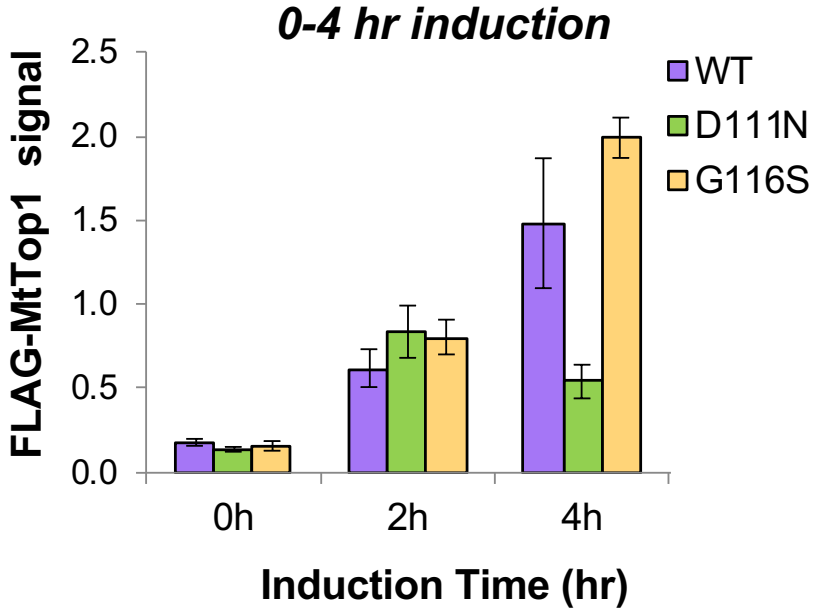

anti-FLAG

Induction Time (hr)

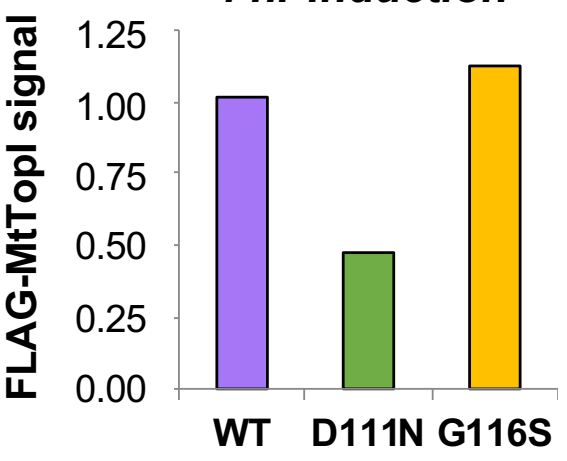

$\mathrm{NaOH}: \quad 0.12 \quad 0.12 \quad 0.25 \quad 0.5 \quad 0.25 \quad 0.25$ kit

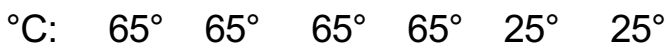

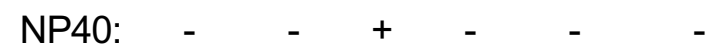

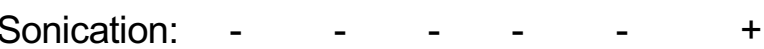

Figure 4 